\title{
Preference, Adherence, and Maintenance of Euthyroidism Using 3 Different Regimens of Levothyroxine Intake during the Fasting Month of Ramadan
}

\author{
Tamer Mohamed Elsherbiny \\ Endocrinology Unit, Alexandria Faculty of Medicine, Alexandria University, Alexandria, Egypt
}

\section{Keywords}

Ramadan · Levothyroxine · Euthyroidism · Adherence .

Preference

\begin{abstract}
Introduction: Millions of Muslim hypothyroid patients fast during Ramadan. Limited data are available on the effect of fasting during Ramadan and different levothyroxine (L-T4) timings on thyroid status. The present study aimed to report preference, adherence, and maintenance of euthyroidism using 3 different regimens of L-T4 intake during Ramadan. Methods: This is a prospective study including Muslim hypothyroid patients fasting during Ramadan between 2018 and 2019. Patients freely chose between 3 regimens, regimen 1 : to take L-T4 at sunset and postpone food and beverages for 60 min; regimen 2: to have iftar (first meal) at sunset, stop food and beverages for 3-4 h, have L-T4, and wait for $60 \mathrm{~min}$ before suhor (last meal); regimen 3: have suhor at midnight, stop food and beverages for $3-4 \mathrm{~h}$, and have L-T4 before next fast. Thyroid status was assessed before and within 6 weeks after Ramadan. Results: 393 patients were included. The first 2 regimens or a combination of both was the most preferred by patients $40.5,36.7$, and $17 \%$, respectively. $323 / 393$ pa-
\end{abstract}

karger@karger.com www.karger.com/dde

Karger $\stackrel{\text { ' }}{5}$

GOPEN ACCESS
(C) 2021 The Author(s)

Published by S. Karger AG, Basel

This article is licensed under the Creative Commons AttributionNonCommercial-NoDerivatives 4.0 International License (CC BYNC-ND) (http://www.karger.com/Services/OpenAccessLicense) Usage and distribution for commercial purposes as well as any distribution of modified material requires written permission. tients were adherent to L-T4 regimens (82.2\%). 273/393 patients were euthyroid after Ramadan (69.5\%). TSH pre- and post-Ramadan were $4.35 \pm 12.30 \mathrm{mIU} / \mathrm{L}$ and $2.73 \pm 3.37$ $\mathrm{mIU} / \mathrm{L}$, respectively, with no statistically significant change $(p=0.225)$. Adherence was predicted post-Ramadan euthyroidism (odds ratio [OR] 2.8 in univariate and OR 2.96 in multivariate models). Conclusions: The first and second regimens or a combination of both was preferred by most patients. High rates of adherence and post-Ramadan euthyroidism were observed. Adherence to the preferred regimen is the main determinant of post-Ramadan euthyroidism.

(c) 2021 The Author(s)

Published by S. Karger AG, Basel

\section{Introduction}

Ramadan - the ninth month of the lunar Islamic calendar - is a holy month for Muslims when adults and adolescents are required to fast - stop food, drinks, and sexual activity from shortly before sunshine to sunset for the whole duration of the month, which ranges between 29 and 30 days [1]. In 2015, the worldwide Muslim population was estimated to be 1.8 billon people [2].

Tamer Mohamed Elsherbiny

Endocrinology Unit, Alexandria Faculty of Medicine, Alexandria University Khartoum Square, Azarita

Alexandria 21521 (Egypt)

tamer_elsherbiny@alexmed.edu.eg 
Hypothyroidism is a common endocrine disease. The estimated prevalence of hypothyroidism in Europe ranges from 4.7 to $4.9 \%$ in the general population, with 80 $83 \%$ of them being subclinically hypothyroid $[3,4]$. The estimated prevalence of hypothyroidism in the USA ranges from 4.6 to $8.9 \%$, with $>90 \%$ of them being subclinically hypothyroid $[5,6]$.

Based on the lowest and highest reported prevalences, projected numbers of Muslims with hypothyroidism would be 82.8 to 160.2 million. Based on the assumption that all overt hypothyroidism patients and at least $24 \%$ of subclinical hypothyroidism patients - with a TSH $>10$ $\mathrm{mIU} / \mathrm{L}$ - may require treatment with levothyroxine (LT4), we can also project that up to 48 million Muslims may require L-T4 therapy [2-6].

Taking levothyroxine (L-T4) 60 min before breakfast was shown to be superior to taking LT-4 with breakfast in 2 studies. Perez et al. [7] and Bach-Huynh et al. [8] both showed that switching L-T4 administration from $60 \mathrm{~min}$ before breakfast to be taken with breakfast significantly raised TSH, resulting in TSH fluctuation in the range from 0 to $13 \mathrm{mIU} / \mathrm{L}$ and from 0 to $19 \mathrm{mIU} / \mathrm{L}$, respectively $[7,8]$. Seechurn et al. [9] reported 10 patients who fulfilled the definition of treatment-refractory hypothyroidism who showed a 40-96\% reduction in TSH levels when switched from breakfast time administration of L-T4 to 60 min before breakfast $[9,10]$.

L-T4 absorption takes place in the small intestine, mainly in the duodenum and jejunum. Having breakfast within 20 min or less after L-T4 means that food will enter the intestine 60-90 min after L-T4 intake. Salvatore et al. [11]reported a subset of hypothyroid patients on L-T4 who present with a delay in the early phase of L-T4 absorption. With this delay, the food reaches the small intestine at a time when $<30 \%$ of L-T4 absorption occurred, so food may sequester LT-4. They found that delaying breakfast for $60 \mathrm{~min}$ after L-T4 will make food enter the small intestine after $2-3 \mathrm{~h}$ from L-T4 administration; at that time, almost $60 \%$ of L-T4 would have been absorbed, with marked improvement of TSH approaching the desired target.

Bedtime administration of L-T4 was compared to 30 min before breakfast - a less than optimum interval for optimal LT-4 absorption - in 2 studies. Bolk et al. [12, 13] in a pilot study of 12 patients and subsequently in a crossover study of 90 patients found that switching the L-T4 intake from $30 \mathrm{~min}$ before breakfast to bedtime, several hours after the last meal of the day, significantly reduced TSH levels from 5.1 to $1.2 \mathrm{mIU} / \mathrm{L}$ in the first and a significant reduction in TSH of $1.25 \mathrm{mIU} / \mathrm{L}$ in the second study.

Preference and Adherence to

Levothyroxine Regimens in Ramadan
However, an opposite result was reported by BachHuynh et al. who found that switching L-T4 from $60 \mathrm{~min}$ before breakfast - an optimal interval for optimal absorption of L-T4 - to bedtime, only $2 \mathrm{~h}$ after the last meal of the day, resulted in a significant increase in TSH from 1.06 to $2.19 \mathrm{mIU} / \mathrm{L}$ [8]. It seems that the interval between the last meal of the day and bedtime administration of L-T4 is the main determinant of TSH, with an interval of 3-4 h after the last meal performing better than $2 \mathrm{~h}$, so we can conclude that a 3- to 4-h interval is the optimal interval.

Based on these observations, both the American Thyroid Association (ATA) and the European Thyroid Association (ETA) recommend that L-T4 be taken after awakening from sleep - on empty stomach by default and $60 \mathrm{~min}$ before breakfast, or for those who have an early evening meal, at least 3-4 h after the last meal and before going to sleep [14-16]. Few studies with a limited number of patients have reported the effect of fasting during Ramadan on thyroid function in Muslim hypothyroid patients treated with L-T4 following L-T4 intake regimens that do not comply with the current recommendations by the ATA and ETA and do not guarantee optimal L-T4 absorption [17-21]. The present study aimed to evaluate preference, adherence, and maintenance - or attainment - of euthyroidism using 3 different regimens of administering L-T4, regimens that fulfill the 2 conditions recommended by the ATA: first, to take the L-T4 tablet at least 3-4 $\mathrm{h}$ after the last meal and second, to take the tablet $60 \mathrm{~min}$ before the next meal or beverages, during the holy month of Ramadan in fasting hypothyroid patients in Egypt.

\section{Methods}

This is a prospective study including Muslim hypothyroid patients willing to fast during Ramadan during the years 2018 and 2019 attending the endocrinology outpatient clinic, Alexandria Faculty of Medicine, Alexandria University, Egypt. The included patients were either stable on L-T4 therapy or initiated L-T4 therapy before the start of Ramadan. The exclusion criteria were thyroid cancer patients requiring L-T4 suppressive therapy, central hypothyroidism, pregnancy, chronic heart failure, liver cirrhosis, renal failure, and acute medical or surgical illness at the time of evaluation to avoid acute and chronic nonthyroidal illness syndromes. All patients were explained the nature and aim of the study and signed an informed written consent. The protocol of the study was approved by the Ethical Committee of the Alexandria Faculty of Medicine (IRB number 12098).

Ramadan: Location, Temperature, and Duration of Fasting

Ramadan experience differs according to the time of the year and latitude of the country in terms of duration of fasting and at- 
mospheric temperature. Because these factors may impact the patients' preference of the regimens under investigation, it was essential to describe the experience of the studied patients. The present study was conducted in Egypt during 2 successive Ramadan months. The first was Ramadan of the Islamic calendar year of 1439 coinciding from May 17 to June 14, 2018. Atmospheric temperatures ranged from 18 to $41^{\circ} \mathrm{C}$, and the duration of fasting ranged from $15 \mathrm{~h}: 02 \mathrm{~min}$ to $15 \mathrm{~h}: 29 \mathrm{~min}$. The second was Ramadan of the Islamic calendar year of 1440 coinciding from May 6 to June 4 , 2019. Atmospheric temperatures ranged from 21 to $44^{\circ} \mathrm{C}$, and the duration of fasting ranged from $14 \mathrm{~h}: 44 \mathrm{~min}$ to $15 \mathrm{~h}: 23 \mathrm{~min}$ [22].

\section{Regimens under Investigation}

The recruited patients were explained 3 regimens for L-T4 intake during Ramadan which comply with the current ATA and ETA recommendations, and they were free to choose any of them according to their preference. Regimen 1 was to take L-T4 tablet(s) at sunset with ample water and start having iftar meal - the first meal after breaking the fast in Ramadan - and beverage(s) after 60 min, and patients were encouraged to perform Maghrib prayers and may read Quran to make good use of this hour. Regimen 2 was to have iftar meal at sunset with beverage(s) and dessert - as is the custom in Egypt - then to stop food and beverages for 3-4 h to guarantee an empty stomach then to take the L-T4 tablet and resume food and beverages $60 \mathrm{~min}$ later and have suhor, the last meal before starting the new fast. Regimen 3 was to have food and beverages - on the patients' will - from sunset till 11 p.m. or midnight and then to stop food and beverages for 3-4 h to guarantee an empty stomach and then to take the L-T4 tablet immediately before starting the fast at sunrise.

Patients were allowed to mix regimens at their will - mixed regimens were assigned numbers from 4 to 7 . Regimen 4 is a mix between regimens 1 and 2, regimen 5 was a mix between regimens 2 and 3, regimen 6 was a mix between regimens 1 and 3, and finally, regimen 7 was a mix of all 3 regimens. Patients were labeled as following a mixed regimen if they followed a second regimen for 2 or more days during the month.

Adherence was assessed by interviewing the participants during post-Ramadan visit. Nonadherence was defined as stopping food and beverages for $<3 \mathrm{~h}$ before taking the L-T4 tablet(s) or stopping food and beverages for $<45 \mathrm{~min}$ after taking the L-T4 tablet(s). The patients who skipped L-T4 tables for 2 or more days without making up for their missed doses were excluded from the study.

Thyroid status was assessed for recruited patients during the pre-Ramadan visit using TSH. Our institution uses electrochemiluminescence immunoassay on Cobas e 411 (Roche Diagnostics $\mathrm{GmbH}$, Mannheim, Germany). The patients were considered euthyroid when TSH was $0.3-4 \mathrm{mIU} / \mathrm{L}$ for patients younger than 70 years and 1-5 mIU/L for patients older than 70 years according to ETA recommendations [15]. Thyroid status was reassessed during the post-Ramadan visit using TSH, provided that this visit comes within 6 weeks from the end of Ramadan. The patients were excluded from the study if the post-Ramadan visit was delayed beyond 6 weeks after Ramadan or if they did not report TSH during the post-Ramadan visit.

\section{Statistical Methods}

Data were analyzed using IBM SPSS software package version 20.0, IBM Corp., Armonk, NY, USA. Comparison between differ-
Table 1. Patient characteristics at inclusion and main outcomes $(n=393)$

\begin{tabular}{|c|c|}
\hline & $N(\%)$ \\
\hline \multicolumn{2}{|l|}{ Sex } \\
\hline Female & $379(96.4)$ \\
\hline Male & $14(3.6)$ \\
\hline \multicolumn{2}{|l|}{ Age, years } \\
\hline Range & $10-73$ \\
\hline Mean & 40.19 \\
\hline \multicolumn{2}{|l|}{ Year of inclusion } \\
\hline 2018 & $147(37.4)$ \\
\hline 2019 & $246(62.6)$ \\
\hline \multicolumn{2}{|l|}{ Etiology of hypothyroidism } \\
\hline Hashimoto thyroiditis & $291(74)$ \\
\hline Thyroid surgery & $66(16.8)$ \\
\hline Radioactive iodine ablation & $9(2)$ \\
\hline Subacute thyroiditis & $21(5.3)$ \\
\hline Postpartum thyroiditis & 7 (1.9) \\
\hline \multicolumn{2}{|l|}{ Preference } \\
\hline Regimen 1 & $159(40.5)$ \\
\hline Regimen 2 & $144(36.7)$ \\
\hline Regimen 3 & $19(4.8)$ \\
\hline Regimen 4 & $67(17)$ \\
\hline \multicolumn{2}{|l|}{ Adherence } \\
\hline Adherent & $323(82.2)$ \\
\hline Nonadherent & $70(17.8)$ \\
\hline \multicolumn{2}{|l|}{ Thyroid status pre-Ramadan } \\
\hline Euthyroid & $249(63.4)$ \\
\hline Dysthyroid & $144(36.6)$ \\
\hline \multicolumn{2}{|l|}{ Thyroid status post-Ramadan } \\
\hline Euthyroid & $273(69.5)$ \\
\hline Dysthyroid & $120(30.5)$ \\
\hline
\end{tabular}

ent groups regarding categorical variables was tested using the $\chi^{2}$ test. For abnormally distributed data, the Kruskal-Wallis test was used to compare the different groups. To compare the different periods, the Wilcoxon signed rank test was applied. To determine if there are differences on a dichotomous dependent variable between 2 related groups, the $\mathrm{McNemar}$ test was used. Significance of the obtained results was judged at the $5 \%$ level.

\section{Results}

\section{Baseline Characteristics}

The study included 393 hypothyroid patients, the majority of whom were female (379 patients [96.4\%]), and only 14 male patients were included in the study (3.6\%). Of the total patients, 147 patients were included in the year $2018(37.4 \%)$, and 246 patients were included in the year 2019 (62.6\%). The age of recruited patients ranged from 10 to 73 years; the mean age of the total patients was 40.19 years, with 13 patients younger than 18 years; and 
Fig. 1. Thyrotropin before and after Ramadan. a TSH before and after Ramadan in the total study sample ( $n=393$ patients) $(p=0.225)$. b TSH before and after Ramadan in patients who were euthyroid at the time of inclusion ( $n=249$ patients) $(p=$ 0.006). Statistically significant at $p \leq 0.05$.

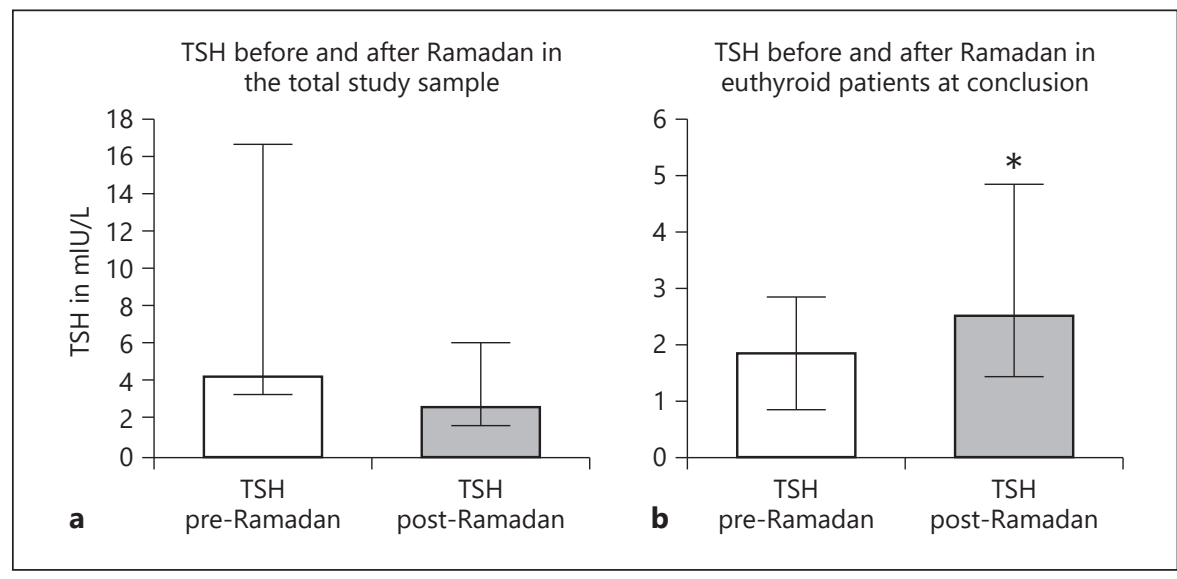

Table 2. Main outcomes in participants euthyroid at inclusion $(n=249)$

\begin{tabular}{lc}
\hline & $N(\%)$ \\
\hline Preference & \\
Regimen 1 & $88(35.3)$ \\
Regimen 2 & $97(39)$ \\
Regimen 3 & $11(4.4)$ \\
Regimen 4 & $49(19.7)$ \\
Adherence & $201(80.7)$ \\
Adherent & $48(19.3)$ \\
Nonadherent & \\
Thyroid status post-Ramadan & $185(74.3)$ \\
Euthyroid & $64(25.7)$ \\
Dysthyroid &
\end{tabular}

9 patients were 70 years of age or older. The etiology of hypothyroidism was as follows: Hashimoto thyroiditis in 291 patients $(74 \%)$, thyroidectomy 66 patients $(16.8 \%)$, De Quervain's thyroiditis 21 patients (5.3\%), radioiodine ablation 9 patients (2\%), and postpartum thyroiditis 7 patients (1.9\%). Regarding the thyroid status, 249 patients were euthyroid $(63.4 \%), 84$ patients were under-replaced (21.4\%), and 60 patients were over-replaced (15.2\%) (Table 1).

\section{Main Outcomes: Preference, Adherence, and Thyroid \\ Status}

Regimen 1 was the most preferred by patients (159 patients, $40.5 \%$ ), followed by regimen 2 (144 patients, $36.7 \%)$, followed by a mix of these 2 regimens, regimen 4 (67 patients, 17\%), followed by regimen 3 (19 patients, $4.8 \%$ ), regimen 5 (2 patients, $0.5 \%$ ), and regimen 7 (2 pa- tients, $0.5 \%)$. Most patients were considered adherent (323 patients, $82.2 \%$ ), and only a minority of patients were considered nonadherent (70 patients, $17.8 \%$ ).

Most patients were euthyroid (273 patients, 69.5\%), 73 patients were under-replaced (18.5\%), and 47 patients were over-replaced (12\%). TSH pre- and post-Ramadan were $4.35 \pm 12.30 \mathrm{mIU} / \mathrm{L}$ and $2.73 \pm 3.37 \mathrm{mIU} / \mathrm{L}$, respectively, showing no statistically significant change $(p=$ 0.225), as shown in Figure 1a.

Main Outcomes in Participants Euthyroid at Inclusion

Among patients who were euthyroid at the time of inclusion - during the pre-Ramadan visit - (249 patients), 88 patients preferred regimen 1 (35.3\%), 97 patients preferred regimen 2 (39\%), 11 patients preferred regimen 3 (4.4\%), 49 patients preferred regimen $4(19.7 \%)$, and 2 patients preferred both regimens 5 and 7 (0.8\%) (Table 2). Most patients were considered adherent (201 patients, $80.7 \%$ ), and only a minority of patients were considered nonadherent (48 patients, 19.3\%).

Most patients were euthyroid (185 patients, $74.3 \%$ ), 39 patients were under-replaced (15.7\%), and 25 patients were over-replaced (10\%).

Among the patients who were euthyroid at the time of inclusion, TSH pre- and post-Ramadan were $1.84 \pm 1.08$ $\mathrm{mIU} / \mathrm{L}$ and $2.51 \pm 2.50 \mathrm{mIU} / \mathrm{L}$, respectively, showing a statistically significant increase $(p=0.006)$, as shown in Figure 1b.

\section{Composite Outcomes}

TSH did not change significantly from pre-Ramadan to post-Ramadan across different L-T4 regimens: $4.90 \pm$ $11.78 \mathrm{mIU} / \mathrm{L}-2.90 \pm 4.13 \mathrm{mIU} / \mathrm{L},[p=0.588]$ in regimen $1,4.09 \pm 15.22 \mathrm{mIU} / \mathrm{L}-2.76 \pm 2.84 \mathrm{mIU} / \mathrm{L},[p=0.789]$ in 
Fig. 2. Adherence in relation to the L-T4 regimen. Rates of adherence/nonadherence according to the preferred regimen of levothyroxine intake during Ramadan $(p=$ $0.005)$. *Statistically significant at $p \leq 0.05$. L-T4, levothyroxine.

Fig. 3. Adherence in relation to thyroid status. a Rates of post-Ramadan euthyroidism/dysthyroidism according to adherence to the L-T4 regimen during Ramadan $(p \leq 0.001)$. b Rates of adherence/nonadherence in extreme patients' profiles: dysthyroid before Ramadan who became euthyroid after Ramadan versus euthyroid before Ramadan who became dysthyroid after Ramadan $(p \leq 0.001)$. *Statistically significant at $p \leq 0.05$. L-T4, levothyroxine.
Rates of adherence/Non-adherence according to preferred regimen of levothyroxine

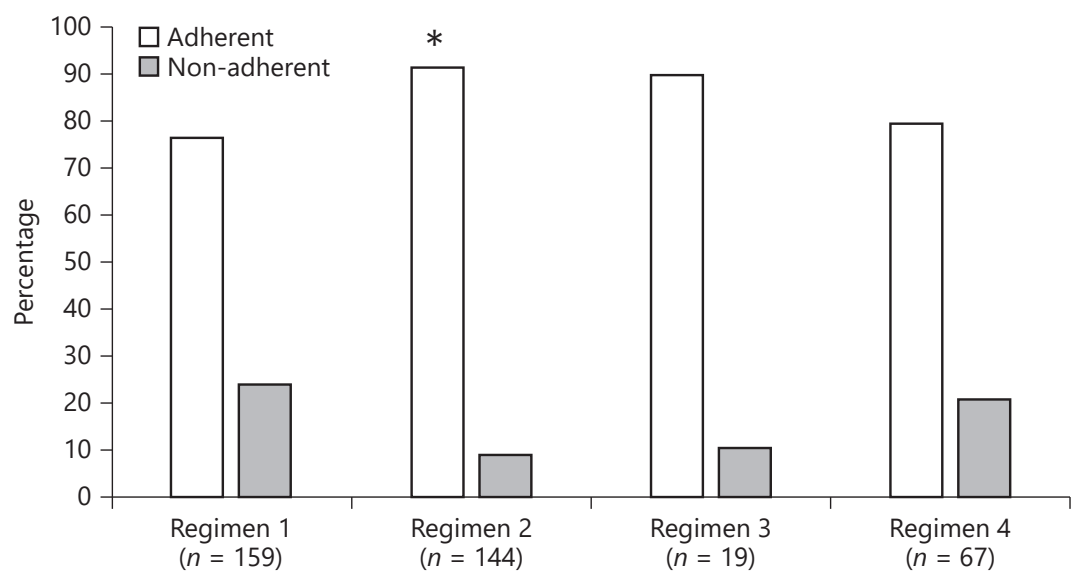

Post-Ramadan thyroid status according to adherence to L-T4

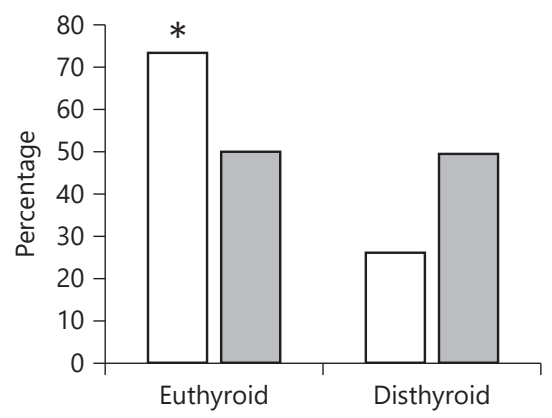

$\square$ Adherent $(n=323)$

$\square$ Non-adherent $(n=70)$

a

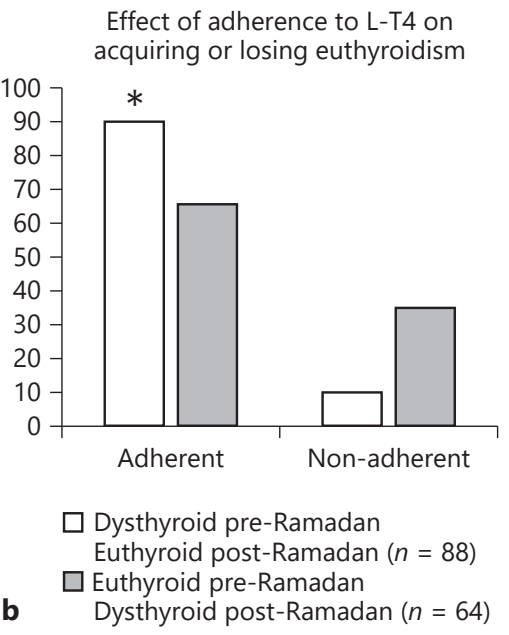

regimen $2,2.15 \pm 2.05 \mathrm{mIU} / \mathrm{L}-2.07 \pm 1.88 \mathrm{mIU} / \mathrm{L},[p=$ 0.658 ] in regimen 3 , and $4.36 \pm 7.77 \mathrm{mIU} / \mathrm{L}-2.37 \pm 2.71$ $\mathrm{mIU} / \mathrm{L}[p=0.091]$ in regimen 4 . The proportion of patients with euthyroidism increased post-Ramadan relative to pre-Ramadan across regimens 1 to 3 , although without statistical significance. Euthyroidism changed from 55.3 to $65.4 \%$ [ $p=0.077]$ in regimen 1 , from $67.4-$ to $72.9 \%$ [ $p=0.341]$ in regimen 2 , and from 57.9 to $68.4 \%$ [ $p=0.727]$ in regimen 3 , with almost no change in regimen $4(73.1-71.6 \%[p=1.000])$.

The regimen preferred by the patients did not predict post-Ramadan thyroid status, neither in the total sample nor in the patients who were euthyroid at the time of inclusion. When classified according to the preferred regi- men, rates of adherence/nonadherence to different regimens were found to be $76.1 / 23.9 \%$ for regimen $1,91 / 9 \%$ for regimen $2,89.5 / 10.5 \%$ for regimen 3, and $79.1 / 20.9 \%$ for regimen 4 . Adherence was significantly higher in regimen $2(91 \%)$ than regimen $1(76.1 \%)(p=0.005)$, as shown in Figure 2.

Statistically significant higher rates of post-Ramadan euthyroidism were observed in adherent patients than in nonadherent patients $(p<0.001)$. Among the adherent patients, 238 patients were euthyroid (73.7\%) and 85 patients were dysthyroid (26.3\%). Among the nonadherent patients, 35 patients were euthyroid (50\%) and 35 patients were dysthyroid (50\%), as shown in Figure 3a.
10 
To further underline the influence of adherence to the L-T4 regimen on post-Ramadan thyroid status, extreme patient profiles were compared based on adherence. The patients who were dysthyroid pre-Ramadan and attained euthyroidism post-Ramadan ( 88 patients, $22.4 \%$ of the total patients) were $89.8 \%$ (79 patients) adherent to L-T4 therapy and only $10.2 \%$ nonadherent ( 9 patients). On the other hand, the patients who were euthyroid pre-Ramadan and became dysthyroid post-Ramadan (64 patients, $16.3 \%$ of the total patients) were only $65.6 \%$ adherent to L-T4 therapy (42 patients) and $34.4 \%$ nonadherent (22 patients). Difference in adherence (89.9 versus 65.6\%) was statistically significant $(p \leq 0.001)$, as shown in Figure $3 b$.

Univariate predictors of post-Ramadan thyroid status were adherence to the preferred regimen ( $[p \leq 0.001]$, odds ratio [OR 2.8] [95\% confidence interval 1.648$4.757])$, pre-Ramadan thyroid status ( $p=0.042)$, and preRamadan TSH $(p=0.007)$. Only adherence to the preferred regimen predicted post-Ramadan thyroid status in multivariate analysis ( $p \leq 0.001]$, [OR 2.963] [95\% confidence interval 1.720-5.103]). Among the patients who were euthyroid at the time of inclusion, only adherence to the preferred regimen predicted post-Ramadan thyroid status ([ $p=0.001]$, [OR 3.2] [95\% confidence interval $1.653-6.209])$.

\section{Discussion}

L-T4 absorption is greatly affected by its relation to food intake [11]. Both the ATA and ETA recommend that for optimal L-T4 absorption, L-T4 should be taken 60 min before breakfast or at bedtime, several hours ( 3 or $4 \mathrm{~h}$ ) after the last meal of the day. [14-16]. No recommendations exist for optimal L-T4 timing during the fasting month of Ramadan [17-21]. Only few studies explored the effect of fasting Ramadan on thyroid status in euthyroid and hypothyroid individuals [23-25].

The present study aimed to report preference, adherence, and maintenance or attainment of euthyroidism using 3 regimens of L-T4 intake - or a combination of these regimens - during the fasting month of Ramadan among hypothyroid Muslim patients. The 3 regimens under investigation were in line with the ATA and ETA evidencebased recommendations regarding a 60 -min interval before the next meal and a several hours (3-4 h) after the last meal to achieve optimal L-T4 absorption [14-16].

To date, only 5 studies aimed to explore the effect of different regimens of L-T4 intake during Ramadan on

Preference and Adherence to

Levothyroxine Regimens in Ramadan thyroid status and TSH levels in hypothyroid fasting Muslim patients shortly after Ramadan. All these studies have been published recently starting from 2013 up to 2020. The included patients range from 47 to 97 patients. Four studies had a prospective design, while the fifth study was a retrospective one [17-21].

The tested timings for L-T4 administration during Ramadan are as follows: $2 \mathrm{~h}$ after meal at bedtime [17], $2 \mathrm{~h}$ after meal and 30 min before the next meal [18], a comparison between $30 \mathrm{~min}$ before iftar versus $30 \mathrm{~min}$ before suhor [19], a comparison between $30 \mathrm{~min}$ before iftar versus $2 \mathrm{~h}$ after iftar versus $1 \mathrm{~h}$ before suhor after $1 \mathrm{~h}$ of empty stomach [20], and finally, a comparison between at iftar versus at suhor [21]. None of these regimens follow the principles of the ATA or ETA evidence-based recommendations. We may conclude that the choice of these regimens or timings was based mainly on what is convenient, feasible, or accepted by the patients, rather than what is physiologic or recommended based on the available literature [17-21].

In the present study, the patients preferred regimens 1 and 2 or a combination of both - regimen 4 - over other regimens. A total of $94.2 \%$ of patients followed these 3 regimens. Patients' preferences were reported in only one study, Or Koca et al. [21] found that $90 \%$ of their patients preferred to take L-T4 at suhor, and only $10 \%$ of them preferred to take it at iftar.

A high rate of adherence to L-T4 regimens was observed in the present study, $82.2 \%$ of the total study population, and $80.7 \%$ of patients who were euthyroid at the time of inclusion were considered adherent. And, 17.8 and $19.3 \%$ were considered nonadherent, respectively. Similar rates of self-reported nonadherence to L-T4 were previously reported in ordinary - non-Ramadan - settings in the range of 21.5 and $22 \%$ [26, 27].

Regarding adherence in Ramadan settings, contrasting results have been reported. Whereas Karoli et al. [17] reported that $75 \%$ of their patients were not adherent to stopping food $2 \mathrm{~h}$ before bedtime L-T4, Sheikh et al. [18] reported the opposite; $75 \%$ of their patients were adherent to L-T4, taking the tables $2 \mathrm{~h}$ after meal and $30 \mathrm{~min}$ before the next meal. Dabbous et al. [19] reported that $70 \%$ of their patients were adherent to $30 \mathrm{~min}$ after iftar and $65 \%$ of their patients were adherent to $30 \mathrm{~min}$ before suhor.

The patients in the present study were empowered to adhere to L-T4 regimens in the pre-Ramadan visit by stressing the importance of adherence to maintain thyroid control, reminding the patients that God forbids Muslims from harming themselves, so if fasting is a duty, 
so is preserving one's health and they must not harm themselves by not adhering to the L-T4 regimen, which may lead to dysthyroidism and, consequently, physical harm. A similar reasoning was recommended by DARIDF diabetes and Ramadan guidelines based on Islamic scholar opinions. This may account for better adherence rates in the present study [28]. Freedom of choice of the regimen may have also contributed to this high adherence.

Post-Ramadan euthyroidism was achieved in 273/393 [69.5\%] of the total study population, $185 / 249$ [74.3\%] of patients who were euthyroid at the time of inclusion, and $238 / 323$ [73.7\%] of patients who were adherent to L-T4 regimens. In the total study population, TSH decreased after Ramadan without statistical significance from 4.35 $\pm 12.30 \mathrm{mIU} / \mathrm{L}$ to $2.73 \pm 3.37 \mathrm{mIU} / \mathrm{L}$. However, when considering the patients who were euthyroid pre-Ramadan, TSH increased significantly after Ramadan from $1.84 \pm 1.08 \mathrm{mIU} / \mathrm{L}$ to $2.51 \pm 2.50 \mathrm{mIU} / \mathrm{L}$.

Karoli et al. [17] reported that $62 \%$ of their patients had an elevation of TSH levels by more than $2 \mathrm{mIU} / \mathrm{L}$ by the end of Ramadan. Likewise, 3 other studies reported a significant increase in mean or median TSH at the end of Ramadan compared to before Ramadan [18, 19, 21].

Fasting Ramadan in euthyroid subjects for an average of $15 \mathrm{~h}$ has been shown to increase TSH within a normal range but significantly relative to pre-Ramadan levels, and it returned to its pre-Ramadan levels around 2 months after Ramadan [23-25]. This interval needed for normalization of TSH coincides with the fact that L-T4 has a long half-life of 7 days, and so, it requires 5 to 6 half-lives to reach a new steady state after the end of the fasting month [16]. That is why we have chosen to evaluate our patients in their post-Ramadan visit within 6 weeks after the end of Ramadan.

Or Koca et al. suggested that L-T4 dose should be increased during Ramadan to prevent dysthyroidism related to fasting based on their results and previous results of Sajid et al. [23] on the effect of fasting on thyroid physiology [21, 23]. However, rates of dysthyroidism among hypothyroid patients on L-T4 therapy in non-Ramadan settings range from 29.5 to $57 \%$, which is very much similar to rates of post-Ramadan dysthyroidism reported in the present study [29], meaning that, in Ramadan, following the regimens that guarantee optimal L-T4 absorption, with high adherence rates, will maintain rates of euthyroidism similar to non-Ramadan settings without modifying L-T4 doses.

The regimen preferred by the patients did not predict post-Ramadan thyroid status nor did it affect post-Rama- dan TSH values. The regimen with the highest adherence was regimen $2(131 / 144$ [91\%]), which was significantly higher than regimen $1(121 / 159$ [76.1\%]).

Adherence to the preferred regimen appears to be the most important determinant of post-Ramadan euthyroidism in the present study. There were significantly higher rates of post-Ramadan euthyroidism among the adherent than nonadherent patients. There were statistically higher rates of adherence among the patient profile dysthyroid pre-Ramadan and euthyroid post-Ramadan (42/64 [89.8\%]) than patient profile euthyroid pre-Ramadan and dysthyroid post-Ramadan (79/88 [65.6\%]). Adherence predicted post-Ramadan euthyroidism in the total sample (OR 2.8 in univariate, OR 2.96 in multivariate models) and in patients who were euthyroid at inclusion (OR 3.2).

The main strengths of the present study are the large number of recruited patients (393 patients); the wide variety of L-T4 regimens formulated according to evidence-based recommendations, which will fit different patient profiles with different needs; freedom of choice of the regimen to follow, rather than assigning patients to regimens unsuitable for their needs; and finally, the inclusion of both euthyroid and dysthyroid patients in a setting similar to daily practice. The main limitation of the present study is difficulty in generalizing the results of this study performed during Ramadan due to variations in the duration of fasting, atmospheric temperature, dietary habits, social standards, and racial differences from country to country and from year to year. Another limitation was assessing adherence by patient interview, rather than using standardized questionnaires. One last limitation is including patients who are both euthyroid and dysthyroid, although this was intended to simulate everyday practice with at least a third of hypothyroid patients attending pre-Ramadan visit with an abnormal TSH; the results were presented both for the whole cohort and for those euthyroid at the time of inclusion.

In conclusion, freedom of choice and adherence to a suitable regimen that complies to evidence-based recommendations of L-T4 intake are crucial to achieving and maintaining post-Ramadan euthyroidism. The first and second regimens or a combination of both was preferred by most patients. High rates of adherence and post-Ramadan euthyroidism were observed in the present study. Adherence to the preferred regimen is the main determinant of post-Ramadan euthyroidism.
12

Dubai Diabetes Endocrinol J 2021;27:6-13 DOI: $10.1159 / 000513927$
Elsherbiny 


\section{Statement of Ethics}

All patients were explained the nature and aim of the study and signed an informed written consent. The protocol of the study was approved by the Ethical Committee of the Alexandria Faculty of Medicine (IRB number 12098).

\section{Conflict of Interest Statement}

The author has no conflicts of interest to declare.

\section{Funding Sources}

This research was funded by the author, and no grant was used to conduct this research.

\section{Author Contributions}

The author is responsible for conception, design, and drafting of the work and acquisition, analysis, and interpretation of data for the work.

\section{References}

1 Ramadan. Encyclopaedia Britannica, Inc. 26 January 2020. https://www.britannica.com/ topic/Ramadan. Accessed $2020 \mathrm{Feb} 08$.

2 Conrad H, Potančoková M, Skirbekk V, Stonawski M, Cooperman A, McClendon D, et al. The changing global religious landscape. Pew Research Center. 2017.

3 Garmendia Madariaga A, Santos Palacios S, Guillén-Grima F, Galofré JC. The incidence and prevalence of thyroid dysfunction in Europe: a meta-analysis. J Clin Endocrinol Metab. 2014;99(3):923-31.

4 Mendes D, Alves C, Silverio N, Batel Marques F. Prevalence of undiagnosed hypothyroidism in Europe: a systematic review and metaanalysis. Eur Thyroid J. 2019;8(3):130-43.

5 Canaris GJ, Manowitz NR, Mayor G, Ridgway EC. The Colorado thyroid disease prevalence study. Arch Intern Med. 2000;160(4):526-34.

6 Hollowell JG, Staehling NW, Flanders WD, Hannon WH, Gunter EW, Spencer CA, et al. Serum TSH, T (4), and thyroid antibodies in the United States population (1988-1994): national health and nutrition examination survey (NHANES III). J Clin Endocrinol Metab. 2002;87(2):489-99.

7 Perez CL, Araki FS, Graf H, de Carvalho GA. Serum thyrotropin levels following levothyroxine administration at breakfast. Thyroid. 2013;23(7):779-84.

8 Bach-Huynh TG, Nayak B, Loh J, Soldin S, Jonklaas J. Timing of levothyroxine administration affects serum thyrotropin concentration. J Clin Endocrinol Metab. 2009;94(10): 3905-12.

9 Seechurn S, Sharma S, Oyibo S. Administration of levothyroxine 45-60 minutes before breakfast improves biochemical availability as evidenced by reduced thyrotropin levels. Open J Endocr Metab Dis. 2012;02(03):36-9.

10 Centanni M, Benvenga S, Sachmechi I. Diagnosis and management of treatment-refractory hypothyroidism: an expert consensus report. J Endocrinol Invest. 2017;40(12):1289301
11 Benvenga S, Bartolone L, Squadrito S, Lo Giudice F, Trimarchi F. Delayed intestinal absorption of levothyroxine. Thyroid. 1995; 5(4):249-53.

12 Bolk N, Visser TJ, Kalsbeek A, van Domburg RT, Berghout A. Effects of evening vs morning thyroxine ingestion on serum thyroid hormone profiles in hypothyroid patients. Clin Endocrinol. 2007;66(1):43-8.

13 Bolk N, Visser TJ, Nijman J, Jongste IJ, Tijssen JG, Berghout A. Effects of evening vs morning levothyroxine intake: a randomized doubleblind crossover trial. Arch Intern Med. 2010; 170(22):1996-2003.

14 Garber JR, Cobin RH, Gharib H, Hennessey JV, Klein I, Mechanick JI, et al. Clinical practice guidelines for hypothyroidism in adults: cosponsored by the American Association of clinical endocrinologists and the American thyroid association. Thyroid. 2012;22(12): 1200-35.

15 Pearce SH, Brabant G, Duntas LH, Monzani F, Peeters RP, Razvi S, et al. 2013 ETA guideline: management of subclinical hypothyroidism. Eur Thyroid J. 2013;2(4):215-28.

16 Jonklaas J, Bianco AC, Bauer AJ, Burman KD, Cappola AR, Celi FS, et al. Guidelines for the treatment of hypothyroidism: prepared by the American thyroid association task force on thyroid hormone replacement. Thyroid. 2014;24(12):1670-751.

17 Karoli R, Fatima J, Chandra A, Mishra PP. Levothyroxine replacement and Ramadan fasting. Indian J Endocrinol Metab. 2013;17(2): 318-9.

18 Sheikh A, Mawani M, Mahar SA. Impact of ramadan fasting on thyroid status and quality of life in patients with primary hypothyroidism: a prospective cohort study from Karachi, Pakistan. Endocr Pract. 2018;24(10):882-8.

19 Dabbous Z, Alowainati B, Darwish S, Ali H, Farook S, Al Malaheem M, et al. A prospective study comparing two-time points of thyroid hormone replacement during the holy month of ramadan. Int J Endocrinol. 2019;2019: 9843961.
20 Zaboon IA, Alidrisi HA, Hussein IH, Alabbood M, Alibrahim NTY, Almomin AMS, et al. Best time for levothyroxine intake in ramadan (THYRAM): basrah experience. Int J Endocrinol Metab. 2020 Apr 6;18(2):e94325.

21 Or Koca A, Dağdeviren M, Altay M. Should the dose of levothyroxine be changed in hypothyroidism patients fasting during Ramadan? Turk J Med Sci. 2020;50(4):784-8.

22 Weather history for Alexandria-Egypt, PA for May/June, 2018/2019 [Accuweather]. Retrieved from: https://www.accuweather.com/ en/eg/alexandria/126995/may-weather/126995. Accessed 2020 Feb 8.

23 Sajid KM, Akhtar M, Malik GQ. Ramadan fasting and thyroid hormone profile. J Pak Med Assoc. 1991;41(9):213-6.

24 Ahmadinejad Z, Ziaee V, Rezaee M, Yarmohammadi L, Shaikh H, Bozorgi F, et al. The effect of ramadan fasting on thyroid hormone profile: a cohort study. Pak J Biol Sci. 2006; 9(10):1999-2002.

25 Bahrayni SH, Vakili V, Nematy N, Norouzy A, Hashemy I, Ebrahimi M. The effect of ramadan fasting on thyroid hormones in 9-13 years old pre-menarche girls. J Fasting Health. 2013;1(2):46-52.

26 Crilly M. Thyroxine adherence in primary hypothyroidism. Lancet. 2004;363(9420):1558.

27 McMillan M, Rotenberg KS, Vora K, Sterman $A B$, Thevathasan L, Ryan MF, et al. Comorbidities, concomitant medications, and diet as factors affecting levothyroxine therapy: results of the control surveillance project. Drugs R D. 2016;16(1):53-68.

28 Eligar V, Taylor PN, Okosieme OE, Leese GP, Dayan CM. Thyroxine replacement: a clinical endocrinologist's viewpoint. Ann Clin Biochem. 2016;53(Pt 4):421-33.

29 Eligar V, Taylor PN, Okosieme OE, Leese GP, Dayan CM. Thyroxine replacement: a clinical endocrinologist's viewpoint. Ann Clin Biochem. 2016;53(Pt 4):421-33.
Preference and Adherence to Levothyroxine Regimens in Ramadan
Dubai Diabetes Endocrinol J 2021;27:6-13 DOI: $10.1159 / 000513927$ 